\title{
Combinatorial Synthesis of Transition Metal Oxide Superlattices
}

\author{
Mikk LiPPMAA, Tsuyoshi OHNISHI, Hideomi KoInuma* ${ }^{*}{ }^{\dagger}$ and Takashi KoIDA* ${ }^{*}, \dagger$ \\ Institute for Solid State Physics, University of Tokyo, 5-1-5 Kashiwanoha, Kashiwa, Chiba 277-8581 \\ * Materials and Structures Laboratory, Tokyo Institute of Technology, \\ 4259 Nagatsuta, Midori-ku, Yokohama, Kanagawa 226-8503
}

(Received April 15, 2004)

\begin{abstract}
We show how the ideas of high-throughput combinatorial synthesis can be translated from organic chemistry to solidstate thin film synthesis. In particular, we describe a combinatorial laser molecular beam epitaxy setup that can synthesize libraries of complex transition-metal oxide lattices and superlattices. As a demonstration of the capabilities of the system, we have analyzed the effects of artifical phase separation in ferromagnetic $\left[\left(\mathrm{LaMnO}_{3}\right)_{m} /\left(\mathrm{SrMnO}_{3}\right)_{m}\right]_{n}$ superlattices.
\end{abstract}

\section{Introduction}

Transition metal oxides have provided an unusually rich playground for materials science and solidstate physics research. The properties of these oxides attract the interest of device designers and physicists alike; the former enticed by characteristics such as large dielectric constants, piezoelectric and ferroelectric effects, superconductivity, colossal magnetoresistance, etc., while the latter concentrate on understanding why and how such a range of different behaviors can appear in otherwise structurally similar compounds.

Most of the dramatic oxide-related discoveries have been driven by experimental work, rather than theoretical predictions. This is perhaps not surprising, considering that many of the more interesting compounds, such as the high-temperature superconductors, contain 4 or more elements, with some of the elements located in several nonequivalent crystallographic sites, e.g. $\mathrm{Cu}$ and $\mathrm{O}$ in $\mathrm{YBa}_{2} \mathrm{Cu}_{3} \mathrm{O}_{7}$. Partial substitutions are often used to modify the properties of parent compounds, increasing to number of elements to 6 or more, e.g. $\mathrm{Hg}_{0.8} \mathrm{Tl}_{0.2} \mathrm{Ba}_{2} \mathrm{Ca}_{2} \mathrm{Cu}_{3} \mathrm{O}_{8.33 .}{ }^{1)}$ Structural complexity can appear even without increasing the number of elements. For example, magnetoplumbite $\mathrm{Ba}_{2} \mathrm{Co}_{2} \mathrm{Fe}_{12} \mathrm{O}_{22}$ has a truly gigantic unit

E-mail: mlippmaa@issp.u-tokyo.ac.jp

$\dagger$ Also at CREST, Japan Science and Technology Corporation.

${ }^{\dagger \dagger}$ Present address: Research Center for Photovoltaics, National Institute of Advanced Industrial Science and Technology, Tsukuba, Ibaraki 305-8568 cell with a $c$-axis length of $43.5 \AA$. $^{2}$

An immediate consequence of the compositional or structural complexity is the difficulty of synthesizing many of the interesting and as yet uncharacterized compositions and crystal phases. One possible solution to this problem is to view these large unit cells as consisting of certain functional or structural blocks. In case of the high- $T_{\mathrm{c}}$ materials, it is convenient to look at the crystal as a stack of basic perovskite units. It is thus possible to draw a direct parallel between the large unit cells of various oxides and superlattices. Indeed, block-by-block growth of oxides has been demonstrated for a variety of compounds. ${ }^{3,4)}$ The thin film growth techniques that are used for such block-by-block growth of thermodynamically stable phases are identical to the methods that are used for superlattice growth. From a purely crystallographic point of view there is no difference between a bulk phase and a superlattice where each individual layer has a thickness of only a few atomic layers. The main difference between bulk phases and superlattices grown by thin film deposition techniques lies in their thermal stability. Bulk phases are nearly always grown close to thermodynamic equilibrium conditions and the phase that actually forms is determined by the thermodynamic stability of the particular crystal structure. In some cases, such as the Ruddlesden-Popper homologous series of perovskites (Fig. 1), many phases have very similar formation energies, which can lead to a multiphase crystal. ${ }^{3)}$

Superlattices, on the other hand, are nearly always grown in a process, which is kinetically controlled 


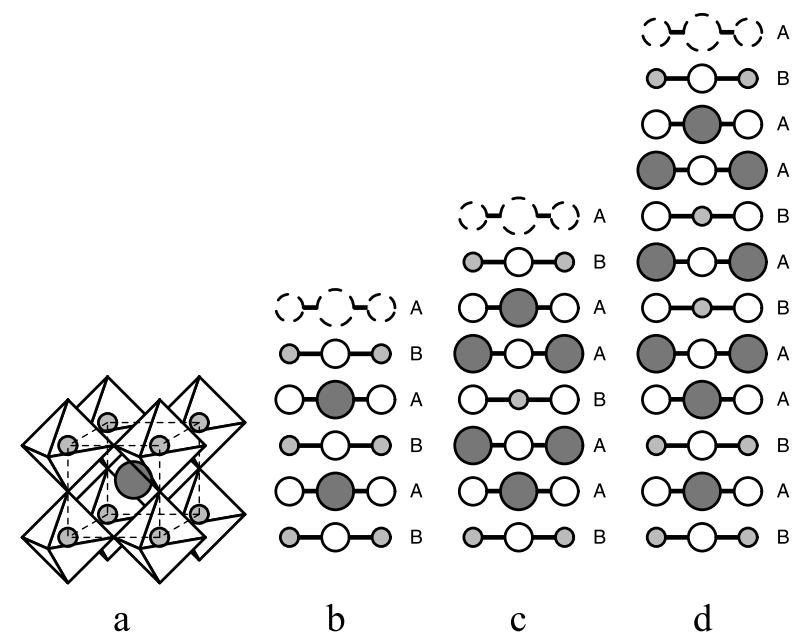

Fig. 1. Ruddlesden-Popper series of perovskites. The basic perovskite unit cell is shown in (a), corresponding to a structure such as $\mathrm{SrTiO}_{3}$. Additional rocksalt-type layers inserted into the lattice give rise to a series of compounds with a general formula of $\mathrm{A}_{n}+{ }_{1} \mathrm{~B}_{n} \mathrm{O}_{3 n+1}$. The structures shown here correspond to $n=\infty$ (a, b), $n=1$ in (c) and $n=2$ in (d).

and the crystal growth front is far from thermodynamic equilibrium, far below the melting point. Adatoms therefore have a very limited ability to diffuse vertically across layer boundaries and it is possible to impose a particular atomic layer ordering on a growing crystal. Perhaps the greatest advantage of superlattice growth is the ability to create artifical materi$\mathrm{als}^{5)}$ that can never be grown in a bulk process.

Unfortunately, the flexibility of thin film superlattice growth as a tool for synthesizing new oxide compositions comes at a price. The atomic layers or blocks that are grown need to maintain charge neutrality and have to be stable on the crystal surface at the growth temperature and ambient gas pressure. Theoretical predictions ${ }^{6}$ ) and experimental observations ${ }^{7)}$ have shown that oxide surface layers can differ significantly from the bulk in terms of atomic positions within an atomic layer, inducing dramatic changes in the electronic structure of the surface layer. This can have a large impact on how the heterointerface forms in an oxide crystal. Even if twodimensional growth can be maintained, there is always a chance of inducing interface states, which would not be present if the same structure would be grown in a bulk process (if that is feasible).

It is clear that layer-by-layer or block-by-block synthesis of complex oxides is an attractive proposition. It is also clear that to succeed, it is necessary to control the growth mode in order to maintain a twodimensional growth front and also to ensure that the heterointerfaces that are formed have the desired atomic and electronic structure.

\section{Combinatorial synthesis}

When the aim is to develop new materials or to optimize the properties of known compounds, it is necessary to test the properties of a large number of samples with different compositions or with different synthesis and processing histories. A similar problem has been solved in the field of organic chemistry by using a parallel synthesis scheme proposed by Merrifield. ${ }^{8,9)}$ Two basic arrangements are commonly used, known as the split-and-mix method and the parallel method, illustrated in Fig. 2. These synthesis methods are particularly effective for creating large numbers of similar molecules that can be built from a limited number basic building blocks, e.g. peptides. In case of split-and-mix synthesis the process starts with a plastic bead, which serves as the starting point for a chain-like molecule. In the process illustarted in Fig. 2 (a), the beads are divided into three samples and the first building blocks are attached to the beads. The reaction results are mixed together and divided into three new samples. Each new sample contains a mixture of molecules consisting of only one block $\left(A_{i}\right)$. In the second step each sample is allowed to react with one element of the second set of building blocks, $B_{j}$, resulting in molecules that all have a length of two blocks and include all possible $A_{i}-B_{j}$ combinations, hence the name, combinatorial synthesis. This process can continue any number of times, producing progressively longer molecules. At the end of the process the plastic beads can be detached and the resulting molecules tested in parallel for specific chemical activity.

Split-and-mix is a very efficient screening method, although even if a certain activity is detected in the mixture, it is not known which particular molecule is responsible. This question can be answered in a parallel synthesis experiemnts where the number of synthesized compounds is usually much smaller, but all compounds are uniquely identifiable. Experiments are based on multiwell plates and highly-automated robotic synthesis and analysis instruments. Combinatorial synthesis and high-throughput screening of synthesis results have now become an established tool in drug design and many other areas of organic chemistry. ${ }^{10)}$

The problem solved by combinatorial synthesis of organic molecules is similar to the issues facing us when designing new inorganic oxides: it is difficult to 


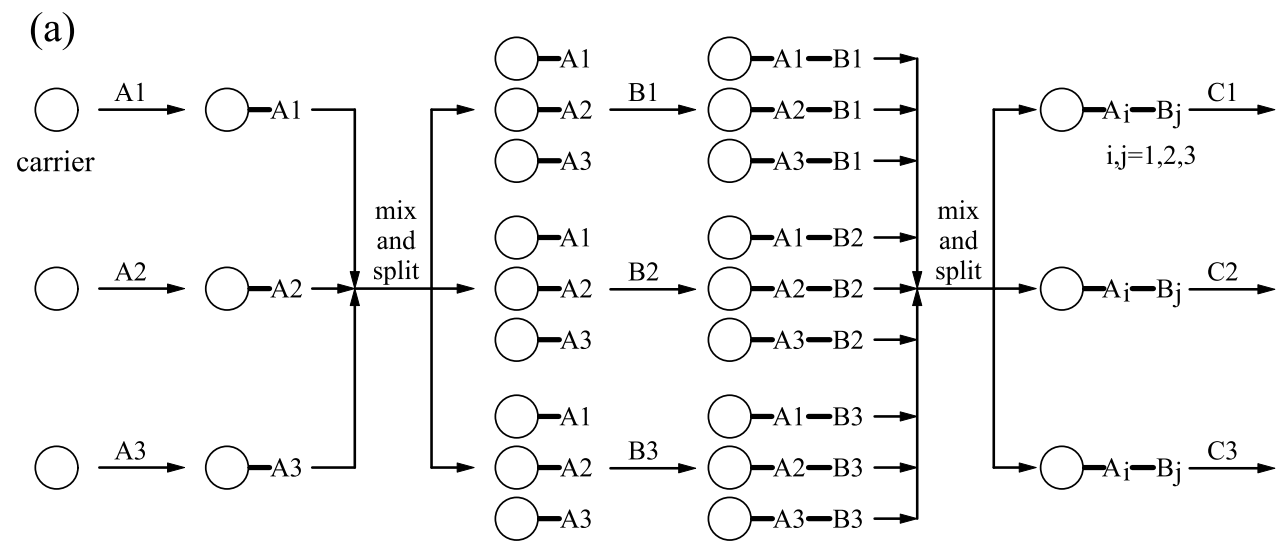

(b)

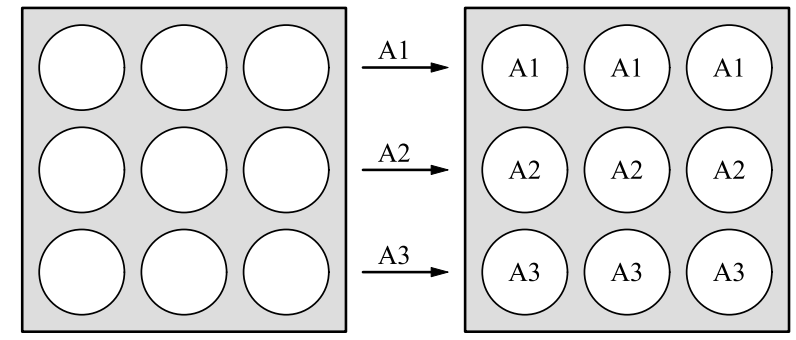

1

2

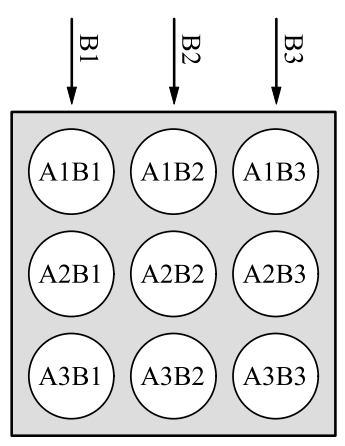

3

Fig. 2. The split-and-mix (a) and parallel (b) combinatorial synthesis methods. The split-and-mix technique is suitable for finding out if a particular functionality exists in a group of compounds. Individual compounds are typically not identifiable. The parallel method (b) can be used to identify within a combinatorial library a particular composition that has the desired functionality.

predict the structure and properties based only on the composition. It is much more efficient to determine the structure and properties once a set of samples has been synthesized. Combinatorial methods have been developed for rapid synthesis ${ }^{11 \sim 13)}$ and characterization $^{14 \sim 16)}$ of a large numbers of solid-state thin film samples.

A comparison of the layered crystal structures of oxides, illustrated in Fig. 1 and the linear molecules produced by combinatorial organic synthesis have obvious conceptual analogies. As shown in Fig. 3, in case of thin film synthesis the multiwell plates can be replaced by a single-crystal substrate. In-situ masking ${ }^{17)}$ can be used to deposit single atomic layers selectively in only certain parts of a larger substrate.

Physically separating individual cells on a single substrate is usually not even necessary, although still possible either by masking or post-deposition processing. The technique can be used to map the properties of different compositions. It is also easy to study the effect of individual layer thicknesses, or to look at how the processing conditions affect the ma-

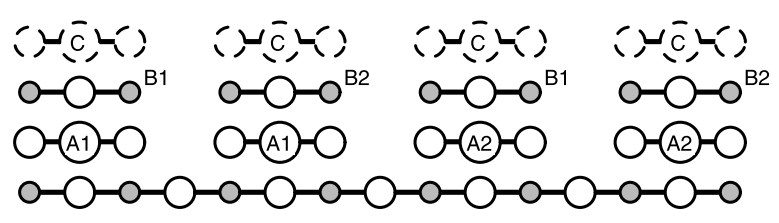

Fig. 3. Combinatorial thin film synthesis. Different samples are grown in parallel in different parts of a single substrate. In situ masking is used to define a unique ordering or thickness of layers for each cell in the combinatorial library.

terial properties or interface formation.

\section{Combinatorial laser MBE}

One of the best techniques for combinatorial oxide thin film growth is laser evaporation. ${ }^{18)}$ The deposition system described here extends the basic laser ablation technique by using a chamber that can work over a wide range of background gas pressures, from $10^{-10}$ to $\approx 1$ Torr. For this reason, the method is often referred to as laser molecular beam epitaxy (LMBE), rather than pulsed laser deposition (PLD), which typically works in the mTorr pressure range. 


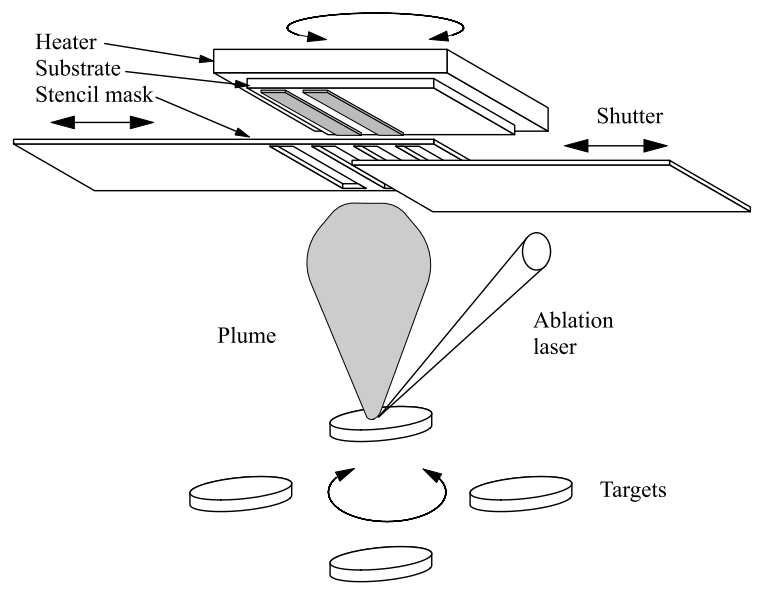

Fig. 4. Diagram of a combinatorial laser molecular beam epitaxy (CLMBE) chamber. The setup includes a heated substrate, a set of movable non-contact masks in front of the substrate and a set of targets that can be ablated in a sequence.

The combinatorial deposition capability is achieved by adding one or more movable non-contact shadow masks just in front of the substrate, as shown in Fig. 4. A laser, typically a $\mathrm{KrF}$ excimer at $248 \mathrm{~nm}$ or a frequency-tripled $\mathrm{Nd}$ : YAG at $352 \mathrm{~nm}$, is used to evaporate material from the surface of single or polycrystalline targets. The evaporated material condenses on the growing film surface. The laser energy density on the target surface is usually in the 1 to 10 $\mathrm{J} / \mathrm{cm}^{2}$ range, which translates to a power density of $200 \mathrm{MW} / \mathrm{cm}^{2}$ to $2 \mathrm{GW} / \mathrm{cm}^{2}$. The main advanatage of the technique is its universality in terms of evaporated materials. The peak power density during a laser pulse is so high, that practically any compound that is solid at room temperature can be ablated with nearly equal yield. This results in a stoichiometric transfer of atoms from the target to the thin film. Polycrystalline targets are usually easy to prepare by conventional powder ceramic techniques. The combinatorial chambers are equipped with target carrousels that can hold from 4 to 12 different targets. All target, mask, and sample motion axes are under computer control, which allows for very complex deposition sequences.

Some of the types of combinatorial samples that such systems can grow are illustrated in Fig. 5. Linear arrays of superlattices (Fig. 5 a) are the easiest to work with, because the growth can be conveniently monitored by reflection high-energy electron diffraction (RHEED). Two-dimensional arrays (Fig. 5 b) are rarely necessary, but can be useful if the characterization probe technique requires a large area, such

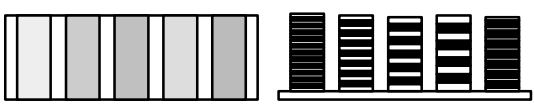

$\mathrm{b}$

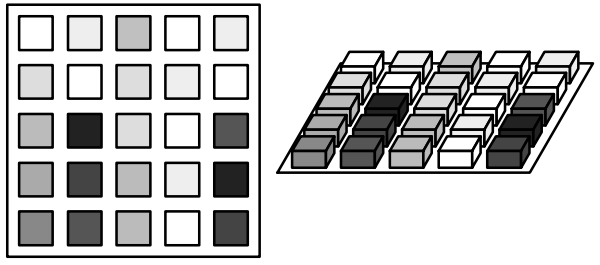

c

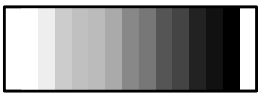

d
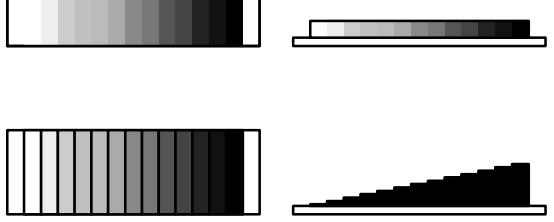

e
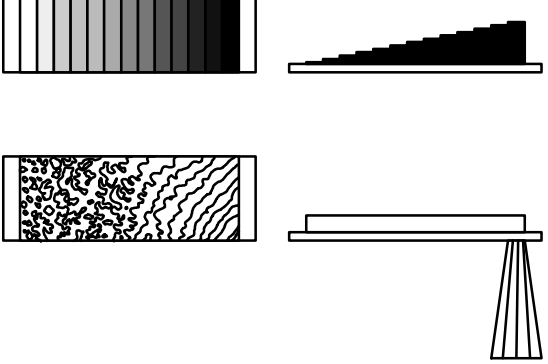

Fig. 5. Typical combinatorial library designs that can be grown by CLMBE: (a) linear superlattice arrays, (b) two-dimensional sample arrays, (c) continuous composition gradients, (d) layer thickness gradients, (e) temperature gradients.

as 4-point transport measurements. Most scanning probe techniques can use composition spread or thickness gardient samples (Figs. $5 \mathrm{c}, \mathrm{d}$ ). Temperature gradient libraries are mostly used to optimize thin film growth conditions.

The size of the libraries and thus the number of samples that can be fabricated in parallel by combinatorial LMBE is limited by the size of the deposition plume. A uniform film thickness can only be achieved in a limited area, typically less than $15 \mathrm{~mm}$ in diameter. In case of oxides, this is not a major limitation because few single crystal substrates are available in sizes greater than that.

\section{Magnetic superlattices}

As an example of what can be achieved with parallel synthesis techniques we describe the growth of artificial $\left[\left(\mathrm{LaMnO}_{3}\right)_{m} /\left(\mathrm{SrMnO}_{3}\right)_{m}\right]_{n}$ superlattices that were used to study the effect of the perovskite A-site cation ordering on the magnetic and transport properties of the colossal magnetoresistance manganites. ${ }^{19)}$

The samples were grown in a CLMBE chamber 


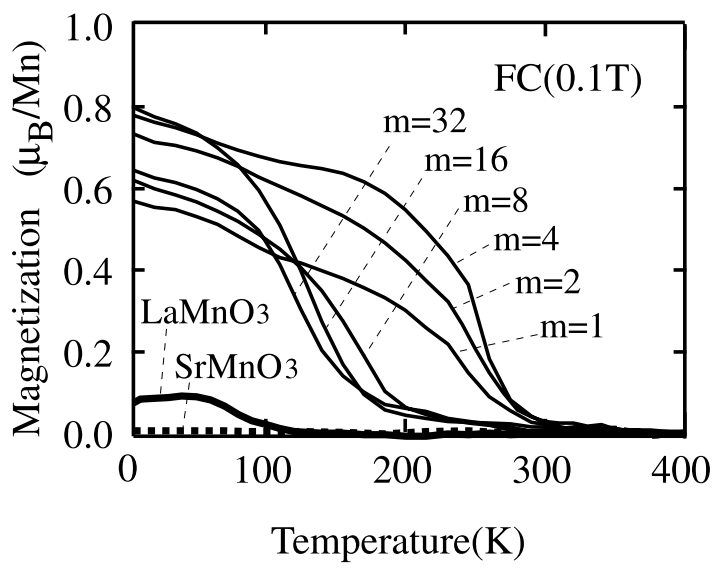

Fig. 6. Magnetization of various $\left[\left(\mathrm{LaMnO}_{3}\right)_{m} /\left(\mathrm{SrMnO}_{3}\right)_{m}\right]_{n}$ superlattices. Reference data for pure $\mathrm{LaMnO}_{3}$ and $\mathrm{SrMnO}_{3}$ films are also shown.

(Fig. 4) that used a stencil mask for separating different superlattice samples on a substrate (Fig. 5 a) and a simple shadow mask for controlling the amount of deposited material in each cell of the library. Each cell was $1.5 \mathrm{~mm}$ wide and 5 different superlattices were grown on a single substrate during each deposition. A $\mathrm{KrF}$ excimer laser was used for ablating ceramic $\mathrm{LaMnO}_{3}$ and $\mathrm{SrMnO}_{3}$ targets in a sequence. The films were grown on atomically smooth $\mathrm{SrTiO}_{3}$ (100) substrates at $800^{\circ} \mathrm{C}$. The background oxygenpressure was $10^{-3}$ Torr. The growth rate was monitored simultaneously at several points on the substrate using a scanning RHEED setup. ${ }^{20)}$ The oxygen pressure was increased to $1 \mathrm{~atm}$. before cooling the films to room temperature. Two libraries were grown, one with superlattice periodicities $m=1,4,16$ and the other with $m=2,8,32$. The total thickness of all superlattices was 96 unit cells. Each library also had reference cells with pure $\mathrm{LaMnO}_{3}$ and $\mathrm{SrMnO}_{3}$ films.

The magnetization of the superlattices as a function of temperature is shown in Fig. 6. Pure $\mathrm{SrMnO}_{3}$ films were completely nonmagnetic, while pure $\mathrm{LaMnO}_{3}$ films showed slight magnetization below $130 \mathrm{~K}$, which coincides with the Néel temperature $T_{N}=135 \mathrm{~K}$ of bulk $\mathrm{LaMnO}_{3}{ }^{21)}$

Superlattices with layer thicknesses $m \leq 4$ showed a systematic increase of magnetization and resistivity (Fig. 7) with the increase of individual layer thicknesses. The Curie temperature was close to $300 \mathrm{~K}$ and the overall magnetic behavior of the lattices was similar to bulk $\mathrm{La}_{1-} \mathrm{Sr}_{x} \mathrm{MnO}_{3}$. The magnetization of thicker lattices, with $m \geq 8$, showed a very different behavior, with a reduced $T_{c}$ and much higher resistivity.

This behavior shows that significant charge trans-

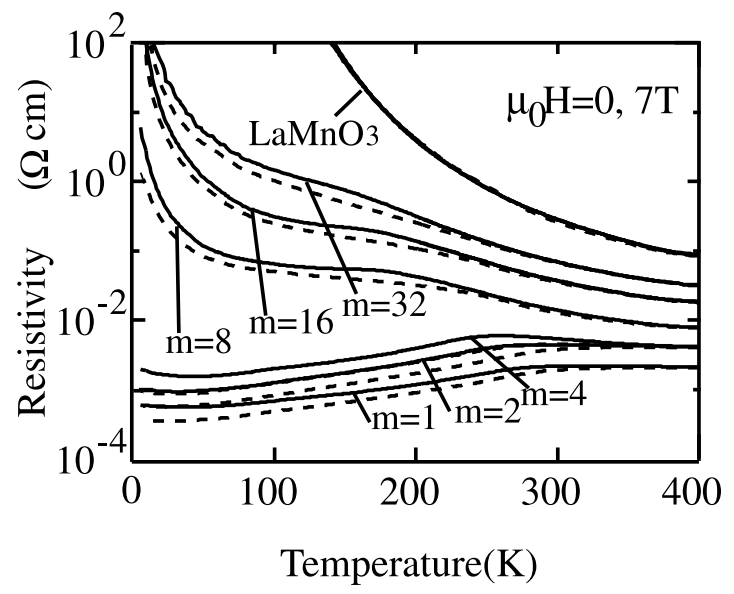

Fig. 7. Resistivity and magnetoresistance of the superlattices as a function of temperature. Measurements are shown for magnetic fields of $0 \mathrm{~T}$ (solid line) and $7 \mathrm{~T}$ (dashed line). A transition from metallic to insulating behavior occurs in the $4<m<8$ layer thickness range.

fer occurs at the $\mathrm{LaMnO}_{3} / \mathrm{SrMnO}_{3}$ interfaces, giving rise to strong ferromagnetism in a superlattice consisting of antiferromagnetic constituent layers. The jump in magnetization that occurs in the $4<m<8$ range shows that the effective layer thickness at the interface that is affected by charge transfer is close to 4 unit cells. When the individual layers are thinner than this, the hole concentration in the lattice is essentially uniform and the crystal behaves as a solid solution $\mathrm{La}_{0.5} \mathrm{Sr}_{0.5} \mathrm{MnO}_{3}$ material would. As the layer thickness increases, the ferromagnetic metallic regions are confined within the $\mathrm{LaMnO}_{3}$ layers, close to the interface with $\mathrm{SrMnO}_{3}$. Due to this, the superlattice periodicity change does not affect the magnetic behavior, but we do see a gradual increase of sample resistivity as the layers became thicker and the volume fraction of conducting interface layers in the superlattice became smaller.

\section{Conclusions}

In conclusion, we have shown that parallel synthesis is an effective tool for exploring the properties of complex oxide lattices. The main advantage is the increased throughput of the experiments, since many samples can be prepared in parallel. In many cases characterization can also be done in parallel, significantly reducing the time that it takes to map out the properties of oxide lattices as the structure or composition is systematically varied. An additional advantage that should not be underestimated is the great increase in the reliability of the data that the experiments yield. Individual film growth experiments al- 
ways have a certain level of variability caused by uncertainties in the thin film deposition and processing conditions. These problems can be largely avoided when experiments are run simultaneously, because all samples are guaranteed to be processed in exactly the same way, and it is possible to integrate reference samples into the same libraries.

\section{References}

1) J. C P Poole, H.A. Farach and R.J. Creswick: "Superconductivity" (Academic Press, San Diego, 1995).

2) I. Ohkubo, Y. Matsumoto, T. Hasegawa, K. Ueno, K. Itaka, P. Ahmet, T. Chikyow, M. Kawasaki and H. Koinuma: Jpn. J. Appl. Phys. 40, L 1343 (2001).

3) J.H. Haeni, C.D. Theis, D.G. Schlom, W. Tian, X.Q. Pan, H. Chang, I. Takeuchi and X.-D. Xiang: Appl. Phys. Lett. 78, 3292 (2001).

4) J.-P. Locquet and E. Mächler: MRS Bulletin 19, 39 (1994).

5) Y. Ogawa, H. Yamada, T. Ogasawara, T. Arima, H. Okamoto, M. Kawasaki and Y. Tokura: Phys. Rev. Lett. 90, 217403 (2003).

6) R. Matzdorf, Z. Fang, Ismail, J. Zhang, T. Kimura, Y. Tokura, K. Terakura and E.W. Plummer: Science 289, 746 (2000).

7) C. Renner, G. Aeppli, B.-G. Kim, Y.-A. Soh and S.-W. Cheong: Nature 416, 518 (2002).

8) R.B. Merrifield: J. Am. Chem. Soc. 85, 2149 (1963).

9) R.B. Merrifield: J. Org. Chem. 29, 3100 (1964).

10) M.J. Plunkett and J.A. Ellman: Sci. Am. 54, April (1997).
11) X.D. Xiang, X. Sung, G. Briceño, Y. Lou, K.A. Wang, H. Chang, W.G. Wallace-Freedman, S.W. Chen and G. Schultz: Science 268, 1738 (1995).

12) H. Koinuma: Solid State Ionics 108, 1 (1998).

13) M. Lippmaa, M. Kawasaki and H. Koinuma: "Combinatorial Materials Synthesis", ed. by X.-D. Xiang and I. Takeuchi (Marcel Dekker, New York, 2003) Chap. 5.

14) T. Fukumura, M. Ohtani, M. Kawasaki, Y. Okimoto, T. Kageyama, T. Koida, T. Hasegawa, Y. Tokura and H. Koinuma: Appl. Phys. Lett. 77, 3426 (2000).

15) T. Ohnishi, D. Komiyama, T. Koida, S. Ohashi, C. Stauter, H. Koinuma, A. Ohtomo, M. Lippmaa, N. Nakagawa, M. Kawasaki, T. Kikuchi and K. Omote: Appl. Phys. Lett. 79, 536 (2001).

16) M. Ohtani, T. Fukumura, M. Kawasaki, K. Omote, T. Kikuchi, J. Harada, A. Ohtomo, M. Lippmaa, T. Ohnishi, D. Komiyama, R. Takahashi, Y. Matsumoto and H. Koinuma: Appl. Phys. Lett. 79, 3594 (2001).

17) M. Lippmaa, T. Koida, H. Minami, Z.W. Jin, M. Kawasaki and H. Koinuma: Appl. Surf. Sci. 189, 205 (2002).

18) H. Koinuma, R. Tsuchiya and M. Kawasaki: SPIE Proc. 2696, 525 (1996).

19) T. Koida, M. Lippmaa, T. Fukumura, K. Itaka, Y. Matsumoto, M. Kawasaki and H. Koinuma: Phys. Rev. B 66, 144418 (2002).

20) H. Koinuma, T. Koida, T. Ohnishi, D. Komiyama, M. Lippmaa and M. Kawasaki: Appl. Phys. A 69[Suppl], S 29 (1999).

21) J. Töpfer and J.B. Goodenough: J. Solid State Chem. 130, 117 (1997). 